OPEN ACCESS

Edited by:

Dumitru Baleanu,

University of Craiova, Romania

Reviewed by:

Francisco Gomez,

Centro Nacional de Investigación y

Desarrollo Tecnológico, Mexico

Kolade Matthew Owolabi

Federal University of Technology,

Nigeria

*Correspondence:

Jagdev Singh

jagdevsinghrathore@gmail.com

Specialty section:

This article was submitted to

Mathematical Physics,

a section of the journal

Frontiers in Physics

Received: 01 October 2018 Accepted: 12 December 2018

Published: 08 January 2019

Citation:

Gill V, Singh J and Singh Y (2019) Analytical Solution of Generalized

Space-Time Fractional

Advection-Dispersion Equation via Coupling of Sumudu and Fourier

Transforms. Front. Phys. 6:151

doi: 10.3389/fphy.2018.00151

\section{Analytical Solution of Generalized Space-Time Fractional Advection-Dispersion Equation via Coupling of Sumudu and Fourier Transforms}

\author{
Vinod Gill ${ }^{1}$, Jagdev Singh ${ }^{2 *}$ and Yudhveer Singh ${ }^{3}$ \\ ${ }^{1}$ Department of Mathematics, Government Post Graduate College Hisar, Hisar, India, ${ }^{2}$ Department of Mathematics, JECRC \\ University, Jaipur, India, ${ }^{3}$ Amity Institute of Information Technology, Amity University, Jaipur, India
}

The objective of this article is to present the computable solution of space-time advection-dispersion equation of fractional order associated with Hilfer-Prabhakar fractional derivative operator as well as fractional Laplace operator. The method followed in deriving the solution is that of joint Sumudu and Fourier transforms. The solution is derived in compact and graceful forms in terms of the generalized Mittag-Leffler function, which is suitable for numerical computation. Some illustration and special cases of main theorem are also discussed.

Keywords: space-time fractional advection-dispersion equation, Fourier transforms, Sumudu transforms, HilferPrabhakar fractional derivative, fractional laplacian operator, Mittag-Leffler function

2010 Mathematics Subject Classification: 26A33, 33E12, 34A08, 42A38, 49K20.

\section{INTRODUCTION}

In the last decade, considerable interest in fractional differential equations has been stimulated due to their numerous applications in the areas of physics, biology, engineering, and other areas. Several numerical and analytical methods have been developed to study the solutions of nonlinear fractional partial differential equations, for details, refer to the work in [1-6]. Fractional equations have enabled the investigation of the nonlocal response of multiple phenomena such as diffusion processes, electrodynamics, fluid flow, elasticity, and many more. Nowadays, fractional derivatives have gained a significant development to model some real life phenomena in the form of partial differential equations or the ordinary equations. Several researchers have performed the numerical simulation for fractional problems and revealed their applications in different directions include [7-12] and references therein. The exchange of heat, mass and momentum are considered to be the fundamental transfer phenomena in the universe. The mathematical framework for heat and mass transfer are of same kind, basically encompass by advection-dispersion equation. In recent work many authors have demonstrated the depth of mathematics and related physical issues of advection-dispersion equations. Schumer et al. [13] gave physical interpretation of space-time fractional advection-dispersion equation. Space-time fractional advection-dispersion equations are generalizations of classical advection-dispersion equations. The use of Hilfer-Prabhakar fractional derivative operator is gaining importance in physics because of their specific properties. The objective of this paper is to derive the solution of Cauchy type generalized fractional advection 
dispersion equation (18), associated with the Hilfer-Prabhakar fractional derivative. This paper provides an elegant extension of results, given earlier by Haung and Liu [14], Haubold et al. [15], Saxena et al. [16], and Agarwal et al. [17].

\section{RESULTS REQUIRED IN THE SEQUEL}

In early 90s, Watugala [18] introduced Sumudu transform, which is defined as,

$$
\begin{aligned}
A= & \left\{f(t) / \exists M, \tau_{i}>0, i=1,2|f(t)| \leq M e^{\frac{|t|}{\tau_{j}}} \text { if } t \in(-1)^{j}\right. \\
& \times[0, \infty)\} .
\end{aligned}
$$

for all real $t \geq 0$ the Sumudu transform of function $f(t) \in A$ is defined as,

$$
S[f(t) ; u]=F(u)=\int_{0}^{\infty} \frac{1}{u} e^{-\frac{t}{u}} f(t) d t, \quad u \in\left(-\tau_{1}, \tau_{2}\right)
$$

inversion formula of (2), is given by

$$
S^{-1}[F(u)]=f(t)=\frac{1}{2 \pi i} \int_{\gamma-i \infty}^{\gamma+i \infty} e^{\frac{t}{u}} F(u) d u,
$$

where $\gamma$ being a fixed real number.

Among others, the Sumudu transform was shown to have units preserving properties, and hence may be used to solve problems without resorting to the frequency domain. Further details and properties about this transform can be found in Belgacem [19], Belgacem et al. [20], and Katatbeh and Belgacem [21].

For a function $u(x, t)$, the Fourier transform of with respect to $x$ is defined by

$$
F[u(x, t)]=u^{*}(\eta, t)=\int_{-\infty}^{\infty} e^{i \eta x} u(x, t) d x, \quad(-\infty<\eta<\infty)
$$

and for the function $u^{*}(\eta, t)$, inverse Fourier transform with respect to $\eta$ is given by the formula

$$
F^{-1}\left[u^{*}(\eta, t)\right]=u(x, t)=\frac{1}{2 \pi} \int_{-\infty}^{\infty} e^{-i \eta x} u^{*}(\eta, t) d \eta
$$

For more details of Fourier transform, see [Debnath and Bhatta [22]].

Mittag-Leffler function of two parameters is studied by Wiman [23] as

$$
E_{\alpha, \beta}(z)=\sum_{n=0}^{\infty} \frac{z^{n}}{\Gamma(\alpha n+\beta)} \quad, \quad \alpha, \beta \in C, R(\alpha)>0 .
$$

Mittag-Leffler function of three parameter introduced by Prabhakar [24] as

$$
E_{\alpha, \beta}^{\gamma}(z)=\sum_{n=0}^{\infty} \frac{\Gamma(\gamma+n)}{\Gamma(\gamma) \Gamma(\alpha n+\beta)} \frac{z^{n}}{n !}, \alpha, \beta, \gamma \in C, R(\alpha)>0 .(7)
$$

Riemann-Liouville fractional integral (right-sided) of order $\alpha$ is defined in [25]

$$
\begin{aligned}
I_{a}^{\alpha}(u(x, t))= & { }_{a}^{R L} D_{t}^{-\alpha}(u(x, t))=\frac{1}{\Gamma(\alpha)} \int_{a}^{t}(t-\tau)^{\alpha-1} u(x, t) d \tau \\
& (t>a), R(\alpha)>0 .
\end{aligned}
$$

The right sided Riemann-Liouville fractional derivative of order $\alpha$ defined as

$$
\begin{aligned}
{ }_{a}^{R L} D_{t}^{\alpha}(u(x, t))= & \left(\frac{d}{d t}\right)^{n}\left(I_{a}^{n-\alpha} u(x, t)\right) \quad(R(\alpha)>0 \\
& n=[R(\alpha)]+1)
\end{aligned}
$$

here $[x]$ is the integral part of $x$.

Caputo [26], introduced fractional derivative of $\operatorname{order} R(\alpha)>$ 0 as

${ }_{0}^{C} D_{t}^{\alpha}(u(x, t))=\left\{\begin{array}{l}\frac{1}{\Gamma(m-\alpha)} \int_{0}^{t} \frac{u^{m}(x, \tau)}{(t-\tau)^{\alpha+1-m}} d \tau, \\ m-1<\alpha \leq m, R(\alpha)>0, m \in N, \\ \frac{\partial^{m}}{\partial t^{m}} u(x, t), \quad \text { if } \alpha=m,\end{array}\right.$

The Sumudu transform of (10) is given in [27], as

$$
\begin{aligned}
S\left[{ }_{0} D_{t}^{\alpha} u(x, t) ; s\right]= & s^{-\alpha} \bar{u}(x, s)-\sum_{k=0}^{m-1} \frac{u^{(k)} u(x, 0)}{u^{\alpha-k}}, \\
& (m-1<\alpha \leq m)
\end{aligned}
$$

where $\bar{u}(x, s)$ is the Sumudu transform of $u(x, t)$.

Hilfer [28], gave a fractional derivative operator of two parameters $\mu$ and $v$, which is generalization of (9) and (10), in the form

$$
\begin{aligned}
{ }_{0} D_{0+}^{\mu, v}(u(x, t))= & I_{t}^{\nu(1-\mu)} \frac{\partial}{\partial t}\left(I_{0+}^{(1-v)(1-\mu)} u(x, t)\right), 0<\mu<1 \\
& \text { and } 0 \leq v \leq 1
\end{aligned}
$$

For $v=0$, equation (12) reduces into (9) and for $v=1$, equation (12) reduces into (10).

The Sumudu transform of (12) is given in [29], as

$$
\begin{aligned}
S\left[{ }_{0} D_{0+}^{\mu, v}(u(x, t)) ; s\right]= & s^{-\alpha} \bar{u}(x, s)-\sum_{k=0}^{m-1} s^{k-m+v(m-\mu)} \frac{\partial^{k}}{\partial x^{k}} \\
& \left(I_{0+}^{(1-v)(1-\mu)} u(x, 0+)\right),
\end{aligned}
$$$$
(m-1<\mu \leq m) .
$$

Where the initial value term $I_{0+}^{(1-v)(1-\mu)} u(x, 0+)$ involves the Riemann-Liouville fractional derivative operator of order $(1-$ v) $(1-\mu)$ as $t \rightarrow 0+$.

A generalization of Hilfer derivate is given in [30], known as Hilfer-Prabhakar derivative, is defined as:

Let $\mu \in(0,1), v \in[0,1]$, and let $f$. belongs to the set of locally integrable real valued functions i.e., $f \in L^{1}[o, b], 0<$ $t<b \leq \infty, f * e_{\rho,(1-v), \omega}^{-\gamma(1-v)}(.) \in A C^{1}[0, b]$. The Hilfer-Prabhakar derivative is defined by

$$
{ }_{0} D_{\rho, \omega .0+}^{\gamma, \mu, \nu}(u(x, t))=E_{\rho, \nu(1-\mu), \omega, 0+}^{-\gamma \nu} \frac{\partial}{\partial t}\left(E_{\rho,(1-\nu)(1-\mu), \omega, 0+}^{-\gamma(1-v)}\right.
$$




$$
u(x, 0+))
$$

where $\gamma, \omega \in R, \rho>0$, and where $E_{\rho, 0, \omega, 0+}^{0} f=f$. We observe that (14) reduces to the Hilfer derivative for $\gamma=0$. The Sumudu transform of this derivative operator (14) is given in [31], in the form:

$$
\begin{aligned}
& S\left[{ }_{0} D_{\rho, \omega, 0+}^{\gamma, \mu, \nu}(u(x, t)) ; s\right]= \\
& s^{-\mu}\left(1-\omega s^{\rho}\right)^{\gamma} \bar{u}(x, s)-s^{\nu(1-\mu)-1}\left(1-\omega s^{\rho}\right)^{\gamma \nu} \\
& {\left[E_{\rho,(1-\nu)(1-\mu), \omega, 0+}^{-\gamma(1-v)}(x, 0+)\right]}
\end{aligned}
$$

For details of this derivative, refer to the work in [30, 31].

Brockmann and Sokolov [32], defined a fractional Laplace operator as:

$$
\Delta^{\frac{\lambda}{2}}=\frac{1}{2 \cos \left(\frac{\pi \lambda}{2}\right)}\left\{-\infty D_{x}^{\lambda}+{ }_{x} D_{\infty}^{\lambda}\right\}, \quad(0<\lambda \leq 2)
$$

where the operators are defined by

${ }_{-\infty} D_{x}^{\lambda}(u(x))=\frac{1}{k-\lambda} \int_{-\infty}^{x} \frac{u^{k}(u)}{(x-u)^{\lambda+1-k}} d u, \quad(k=[\lambda]+1)$,

and

${ }_{x} D_{\infty}^{\lambda}(u(x))=\frac{1}{k-\lambda} \int_{x}^{\infty} \frac{u^{k}(u)}{(x-u)^{\lambda+1-k}} d u, \quad(k=[\lambda]+1)$.

The Fourier transform of $\Delta^{\frac{\lambda}{2}}$ is given in [32], as

$$
F\left\{\Delta^{\frac{\lambda}{2}}(u(x, t)) ; k\right\}=-|k|^{\lambda} F\{u(x, t)\}, \quad(0<\lambda \leq 2) .
$$

Inverse Sumudu transform of the following function is directly applicable in this sequel:

In the complex plane $\mathrm{C}$, for any $R(\alpha)>0, R(\beta)>0$, and $\omega \in C$

$$
S^{-1}\left[u^{\gamma-1}\left(1-\omega u^{\beta}\right)^{-\delta}\right]=t^{\gamma-1} E_{\beta, \gamma}^{\delta}\left(\omega t^{\beta}\right) .
$$

\section{SPACE-TIME FRACTIONAL ADVECTION-DISPERSION EQUATION}

Here we will find, the solution of the generalized space-time Advection-Dispersion equation (18) under the conditions given in (19) and (20). Our main findings in the form of the following Theorem 3.1 and Corollary 3.2.

Theorem 3.1. Consider the generalized fractional order spacetime advection-dispersion equation of Cauchy type

$$
{ }_{0} D_{\rho, \omega, t}^{\gamma, \mu, v}(u(x, t))=-\eta D_{x} u(x, t)+\varsigma \Delta^{\frac{\lambda}{2}}(u(x, t)),
$$

where $\lambda \in(0,2] x \in R, t \in R^{+}, \mu \in(0,1), v \in[0,1]$, with initial condition,

$$
E_{\rho,(1-v)(1-\mu), \omega, 0+}^{-\gamma(1-v)} u(x, 0+)=g(x), \gamma, \omega, x \in R, \rho>0,
$$

and boundary condition

$$
\lim _{|x| \rightarrow \infty} u(x, t)=0, t>0
$$

where $\Delta^{\frac{\lambda}{2}}$ is the Laplace operator of fractional order $\lambda, \lambda \in$ $(0,2]$. The positive constant $\eta$ represent the average fluid velocity and $\zeta$ (positive constant) represent the dispersion coefficient. Subject to the above constraints, solution of equation (18), is

$$
\begin{aligned}
u(x, t)= & \sum_{n=0}^{\infty} \frac{t^{\nu(1-\mu)+n \mu-1}}{2 \pi} \int_{-\infty}^{\infty} e^{-i k x} g(k)\left(i \eta k-\varsigma|k|^{\lambda}\right)^{n} \\
& E_{\rho, \nu(1-\mu)+n \mu}^{\gamma(n-v)}\left(\omega t^{\rho}\right) d k .
\end{aligned}
$$

Proof: First, take the Fourier transform of equation (18) with respect to the space variable $x$, then

$$
{ }_{0} D_{\rho, \omega, t}^{\gamma, \mu, v}\left(u^{*}(k, t)\right)=\eta i k u^{*}(k, t)-\varsigma|k|^{\lambda} u^{*}(k, t),
$$

$u^{*}(k, t)$ represent Fourier transform of $u(x, t)$. Again, apply Sumudu transform on (22) with respect to time variable $t$, we get

$$
\begin{aligned}
& s^{-\mu}\left(1-\omega s^{\rho}\right)^{\gamma \overline{u^{*}}}(k, s)-s^{\nu(1-\mu)-1}\left(1-\omega s^{\rho}\right)^{\gamma \nu} \\
& {\left[E_{\rho,(1-v)(1-\mu), \omega, 0+}^{-\gamma(1-v)} u(k, 0+)\right]=i \eta k \overline{u^{*}}(k, s)-s|k|^{\lambda} \overline{u^{*}}(k, s),}
\end{aligned}
$$

where $S[u(k, t) ; s]=\bar{u}(k, s)$.

Solve equation (23), by using conditions (19)-(20), we get

$$
\begin{aligned}
& \left\{s^{-\mu}\left(1-\omega s^{\rho}\right)^{\gamma}-i \eta k+\varsigma|k|^{\lambda}\right\} \overline{u^{*}}(k, s)=s^{\nu(1-\mu)-1} \\
& \quad\left(1-\omega s^{\rho}\right)^{\gamma \nu} g(k), \\
& \Rightarrow \overline{u^{*}}(k, s)=\frac{s^{\nu(1-\mu)-1}\left(1-\omega s^{\rho}\right)^{\gamma \nu}}{\left\{s^{-\mu}\left(1-\omega s^{\rho}\right)^{\gamma}-i \eta k+\varsigma|k|^{\lambda}\right\}^{\prime}} g(k) .
\end{aligned}
$$

On taking inverse Sumudu transform of equation (24), and after little simplification, apply result (17), it gives

$$
\begin{aligned}
u^{*}(k, t)= & \sum_{n=0}^{\infty}\left(i \eta k-\varsigma|k|^{\lambda}\right)^{n} g(k) t^{\nu(1-\mu)+n \mu-1} \\
& E_{\rho, \nu(1-\mu)+n \mu}^{\gamma(n-\nu)}\left(\omega t^{\rho}\right) .
\end{aligned}
$$

Taking inverse Fourier transform of (25), get our required result (21).

This completes the proof of the theorem 3.1.

0n taking $\eta=0, \varsigma=\frac{i h}{2 m}$ in Theorem 3.1, we arrive at:

Corollary 3.2. Consider the following one dimensional spacetime Schrödinger equation of fractional order, for a free nature particle of mass $m$ is

$$
{ }_{0} D_{\rho, \omega, t}^{\gamma, \mu, v}(u(x, t))=\frac{i h}{2 m} \Delta^{\frac{\lambda}{2}}(u(x, t)),
$$

where $\lambda \in(0,2], x \in R, t \in R^{+}, \mu \in(0,1), v \in[0,1]$, with initial condition

$$
E_{\rho,(1-\nu)(1-\mu), \omega, 0+}^{-\gamma(1-v)} u(x, 0+)=g(x), \quad \gamma, \omega \in R, \rho>0,
$$


and boundary condition

$$
\lim _{|x| \rightarrow \infty} u(x, t)=0, t>0,
$$

where $\Delta^{\frac{\lambda}{2}}$ is same as we defined earlier and $h=6.625 \times$ $10^{-27}$ erg $s=4.21 \times 10^{-21} \mathrm{Mev} s$ is the Planck constant. Subject to the above constraints, solution of equation (26), is

$$
\begin{aligned}
u(x, t)= & \sum_{n=0}^{\infty} \frac{t^{\nu(1-\mu)+n \mu-1}}{2 \pi} \int_{-\infty}^{\infty} e^{-i k x} g(k)\left(-\frac{i h}{2 m}|k|^{\lambda}\right)^{n} \\
& E_{\rho, v(1-\mu)+n \mu}^{\gamma(n-v)}\left(\omega t^{\rho}\right) d k .
\end{aligned}
$$

Proof: For obtaining the solution of Corollary 3.2, we follow same procedure, as we used in the proof of Theorem 3.1, and after little simplification, finally we obtain the desired result (29).

\section{ILLUSTRATION}

Example 4.1. To describe solute transport in aquifers, consider the following generalized fractional advection dispersion equation

$$
{ }_{0} D_{\rho, \omega, t}^{\gamma, \mu, \nu}(u(x, t))=-D_{x} u(x, t)+u^{\prime} \Delta^{\frac{\lambda}{2}}(u(x, t)),
$$

with initial condition

$$
E_{\rho,(1-\nu)(1-\mu), \omega, 0+}^{-\gamma(1-v)} u(x, 0+)=e^{-x}, 0<x<1, t>0,
$$

and boundary condition

$$
\lim _{|x| \rightarrow \infty} u(x, t)=0, t>0,
$$

where $\mu^{\prime}=\frac{d}{v^{\prime} L}$ and we consider a dimensionless parameter, called Peclet number, $P e=\frac{1}{\mu^{\prime}}$ where $L$ is the packing length. The Peclet number determines the nature of the problem, that is, the Peclet number is low for dispersion-dominated problems and is large for advective dominated problems, $d$ is the dispersion coefficient $\left[L^{2} T^{-1}\right]$ and $v^{\prime}$ is the Darcy velocity $\left[L T^{-1}\right]$.

Our interest is in the solution of (30), for this we follow same procedure, as we applied in the proof of Theorem 3.1, and after little simplification, finally we obtain

$$
\begin{aligned}
u(x, t)= & \sum_{n=0}^{\infty} \frac{t^{\nu(1-\mu)+n \mu-1}}{2 \pi} \int_{-\infty}^{\infty} e^{-i k x} g(k)\left(i k-\mu^{\prime}|k|^{\lambda}\right)^{n} \\
& E_{\rho, v(1-\mu)+n \mu}^{\gamma(n-\nu)}\left(\omega t^{\rho}\right) d k
\end{aligned}
$$

Here $u(x, t)$ represent the analytical expression of solute concentration and $g(k)=\frac{1}{\sqrt{2 \pi}}\left[\frac{e^{-(1+i k)}-1}{1+i k}\right]$.

Example 4.2. Consider the generalized fractional order spacetime advection-dispersion equation

$$
{ }_{0} D_{\rho, \omega, t}^{\gamma, \mu, \nu}(u(x, t))=-D_{x} u(x, t)+u^{\prime} \Delta^{\frac{\lambda}{2}}(u(x, t)),
$$

with the initial condition

$$
E_{\rho,(1-v)(1-\mu), \omega, 0+}^{-\gamma(1-v)} u(x, 0+)=\delta(x),
$$

Here $\delta(x)$ is Dirac-delta function and boundary condition

$$
\lim _{|x| \rightarrow \infty} u(x, t)=0, t>0,
$$

The solution of (34) can be obtained by same technique as we applied in proof of Theorem 3.1

$$
\begin{aligned}
u(x, t)= & \sum_{n=0}^{\infty} \frac{t^{\nu(1-\mu)+n \mu-1}}{2 \pi} \int_{-\infty}^{\infty} e^{-i k x}\left(i k-\mu^{\prime}|k|^{\lambda}\right)^{n} \\
& E_{\rho, v(1-\mu)+n \mu}^{\gamma(n-\nu)}\left(\omega t^{\rho}\right) d k .
\end{aligned}
$$

\section{SPECIAL CASES}

Some interesting special cases of Theorem 3.1 are enumerated below:

If we set $\gamma=0$, in (14), then Hilfer-Prabhakar derivative reduces to Hilfer derivative (12), and the Theorem 3.1 reduces to:

(I). Consider the generalized fractional order space-time advection-dispersion equation of Cauchy type

$$
{ }_{0} D_{t}^{\mu, v}(u(x, t))=-\eta D_{x} u(x, t)+\varsigma \Delta^{\frac{\lambda}{2}}(u(x, t)),
$$

where $(0<\lambda \leq 2), x \in R, t \in R^{+}, \mu \in(0,1), v \in[0,1]$, with initial condition

$$
I_{0+}^{(1-v)(1-\mu)} u(x, 0+)=g(x), x \in R,
$$

and boundary condition

$$
\lim _{|x| \rightarrow \infty} u(x, t)=0, t>0 .
$$

For obtaining the solution of (38), follow same procedure as we used in the proof of theorem 3.1, and use (13), after little simplification, obtain the following

$$
\begin{aligned}
u(x, t)= & \frac{t^{\nu(1-\mu)+\mu-1}}{2 \pi} \int_{-\infty}^{\infty} e^{-i k x} g(k) E_{\mu, v(1-\mu)+\mu}^{1} \\
& \left(\left(i \eta k-\varsigma|k|^{\lambda}\right) t^{\mu}\right) d k .
\end{aligned}
$$

Again, use convolution theorem of the Fourier transform to (41), then we get solution of (38), in term of Green's function as

$$
u(x, t)=\int_{-\infty}^{\infty} G(x-k, t) g(k) d k .
$$

Here Green's function is given as

$$
\begin{aligned}
G(x, t)= & \frac{t^{\nu(1-\mu)+\mu-1}}{2 \pi} \int_{-\infty}^{\infty} e^{-i k x} E_{\mu, \nu(1-\mu)+\mu}^{1} \\
& \left(\left(i \eta k-\varsigma|k|^{\lambda}\right) t^{\mu}\right) d k
\end{aligned}
$$

If we set $v=1$ in (12), then Hilfer fractional derivative reduces to Caputo fractional derivative operator (10) and the equation (38), yields the following:

(II). Consider the generalized fractional order space-time advection-dispersion equation of Cauchy type 


$$
{ }_{0} D_{t}^{\mu}(u(x, t))=-\eta D_{x} u(x, t)+\varsigma \Delta^{\frac{\lambda}{2}}(u(x, t)),
$$

where $(0<\lambda \leq 2), x \in R, t \in R^{+}, \mu \in(0,1)$, with initial condition

$$
u(x, 0+)=g(x), x \in R,
$$

and boundary condition

$$
\lim _{|x| \rightarrow \infty} u(x, t)=0, t>0 .
$$

For obtaining the solution of (42), follow same procedure as we used in the proof of theorem 3.1, and use (11), after little simplification, obtain the following

$$
u(x, t)=\frac{1}{2 \pi} \int_{-\infty}^{\infty} e^{-i k x} g(k) E_{\mu, 1}^{1}\left(\left(i \eta k-\varsigma|k|^{\lambda}\right) t^{\mu}\right) d k
$$

Again, use convolution theorem of the Fourier transform to (45) then we get solution of (42), in term of Green's function as

$$
u(x, t)=\int_{-\infty}^{\infty} G(x-k, t) g(k) d k
$$

Here Green's function is given as

$$
G(x, t)=\frac{1}{2 \pi} \int_{-\infty}^{\infty} e^{-i k x} E_{\mu, 1}^{1}\left(\left(i \eta k-\varsigma|k|^{\lambda}\right) t^{\mu}\right) d k
$$

\section{REFERENCES}

1. Atangana A, Gómez-Aguilar JF. Numerical approximation of RiemannLiouville definition of fractional derivative: from Riemann-Liouville to Atangana-Baleanu. Numerical Methods for Partial Differential Equations (2018) 34:1502-23. doi: 10.1002/num.22195

2. Gill V, Modi K, Singh Y. Analytic solutions of fractional differential equation associated with RLC electrical circuit. J Stat Manage Syst. (2018) 21:575-82. doi: 10.1080/09720510.2018.1466966

3. Gómez-Aguilar JF, Yépez-Martínez H, Torres-Jimenez J, Cordova-Fraga T, Escobar-Jimenez RF, Olivares-Peregrino VH. Homotopy perturbation transform method for nonlinear differential equations involving to fractional operator with exponential kernel. Adv Diff Equations (2017) 2017:68. doi: 10.1186/s13662-017-1120-7,1-18

4. Morales-Delgado VF, Gómez-Aguilar JF, Yépez-Martínez H, Baleanu D, Escobar-Jimenez RF, Olivares-Peregrino VH. Laplace homotopy analysis method for solving linear partial differential equations using a fractional derivative with and without kernel singular. Adv Diff Eq. (2016) 2016:164. doi: 10.1186/s13662-016-0891-6,1-17

5. Yépez-Martínez H, Gómez-Aguilar JF, Sosa $a$ IO, Reyesa JM, Torres- Jimenez $\mathrm{J}$. The Feng's first integral method applied to the nonlinear $\mathrm{mKdV}$ space-time fractional partial differential equation. Rev Mex Fís. (2016) 62:310-6.

6. Yépez-Martínez H, Gómez-Aguilar JF. Numerical and analytical solutions of nonlinear differential equations involving fractional operators with power and Mittag-Leffler kernel. Math Modell Nat Phenomena (2018) 13:13. doi: $10.1051 / \mathrm{mmnp} / 2018002$

7. Kumar D, Singh J, Baleanu D. A new analysis of Fornberg-Whitham equation pertaining to a fractional derivative with Mittag-Leffler type kernel. Eur J Phys Plus (2018) 133:70. doi: 10.1140/epjp/i2018-11934-y
(III). On giving suitable value to the parameters involved in Theorem 3.1, we can obtained same results, earlier given by Haung and Liu [14], Haubold et al. [15], Saxena et al. [16], and Agarwal et al. [17].

\section{CONCLUSION}

In this paper, we have presented a solution of generalized spacetime fractional advection-dispersion equation. The solution has been developed in terms of Mittag-Leffler function with the help of Sumudu transform and Fourier transform. We can develop the efficient numerical techniques to find solution of various fractional partial differential equations arising in various fields by considering these analytic solutions as base. For future research, the methodology presented in this paper can serve as a good working template to solve any fractional advection-dispersion equations in higher dimensions.

\section{AUTHOR CONTRIBUTIONS}

VG, JS, and YS designed the study, developed the methodology, collected the data, performed the analysis, and wrote the manuscript.

\section{ACKNOWLEDGMENTS}

The authors are grateful to referees for their suggestions and useful comments on this paper.

8. Owolabi KM. Mathematical analysis and numerical simulation of patterns in fractional and classical reaction-diffusion systems. Chaos Solitons Fractals (2016) 93:89-98. doi: 10.1016/j.chaos.2016.10.005

9. Owolabi KM. Robust and adaptive techniques for numerical simulation of nonlinear partial differential equations of fractional order. Commun Nonlinear Sci Num Simulat. (2017) 44:304-17. doi: 10.1016/j.cnsns.2016.08.021

10. Owolabi KM, Atangana A. Analysis of mathematics and numerical pattern formation in superdiffusive fractional multicomponent system. Adv Appl Math Mech. (2017) 9:1438-60. doi: 10.4208/aamm.OA-2016-0115

11. Kumar D, Singh J, Baleanu D, Rathore S. Analysis of a fractional model of Ambartsumian equation, Eur J Phys Plus (2018) 133:259. doi: 10.1140/epjp/i2018-12081-3

12. Singh J, Kumar D, Hammouch Z, Atangana A. A fractional epidemiological model for computer viruses pertaining to a new fractional derivative. Appl Math Comput. (2018) 316:504-15. doi: 10.1016/j.amc.2017. 08.048

13. Schumer R, Benson DA, Meerschaert M, Wheatcraft SW. Eulerian derivation of the fractional advection-dispersion equation. J Contam Hydrol. (2001) 48:69-88. doi: 10.1016/S0169-7722(00)00170-4

14. Haung F, Liu F. The fundamental solution of the space-time fractional advection- disperssion equation. J Appl Math Comput. (2015) 18:339-50. doi: 10.1007/BF02936577

15. Haubold HJ, Mathai AM, Saxena RK. Solution of reaction-diffusion equations in terms of the H- function. Bull Astro Soc India (2007) 35: 681-9.

16. Saxena RK, Saxena R, Kalla SL. Solution of the space-time fractional Schro dinger equation occurring in quantum mechanics. Fract Calc Appl Anal. (2010) 13:177-99. 
17. Agarwal R, Jain S, Agarwal RP. Analytic solution of generalized space time advection-dispersion equation with fractional Laplace operator. J Nonlinear Sci Appl. (2016) 9:3445-54. doi: 10.22436/jnsa.009.06.09

18. Watugala GK. Sumudu Transform: a new integral transform to solve differential equations and control engineering problems. Int J Math Educ Sci Technol. (1993) 24:35-43. doi: 10.1080/0020739930240105

19. Belgacem FBM. chapter 6, Applications of the sumudu transform to indefinite periodic parabolic equations. In: Proceedings of the $6^{\text {th }}$ International Conference on Mathematical Problems and Aerospace Sciences (ICNPAA '06), Cambridge: Cambridge Scientific (2007). pp. 51-60.

20. Belgacem FBM, Karaballi AA, Kalla SL. Analytical investigations of the sumudu transform and applications to integral production equations. Math Problem Eng. (2003) 2003:103-18. doi: 10.1155/S1024123X03207018

21. Katatbeh QD, Belgacem FBM. Applications of the Sumudu transform to fractional differential equations. Nonlinear Studies (2011) 18:99-112.

22. Debnath L, Bhatta D. Integral Transforms and Their Applications. Boca Raton, FL: CRC Press (1995).

23. Wiman A. ber den Fundamentals in der Teorie der Funktionen $E^{a}(x)$, (German). Acta Math. (1905) 29:191-201.

24. Prabhakar TR. A singular integral equation with a generalized Mittag-Leffler function in the kernel. Yokohama Math J. (1971) 19:7-15.

25. Samko SG, Kilbas AA, Marichev OI. Fractional Integrals and DerivativesTheory and Applications. Linghorne: CRC Press (1993).

26. Caputo M. Elasticita e dissipazione. Bologana: Zani-chelli (1969).

27. Chaurasia VBL, Singh J. Application of sumudu transform in Schrö dinger equation occurring in quantum mechanics. Appl Math Sci. (2010) 4:2843-50.
28. Hilfer R. Applications of Fractional Calculus in Physics. Singapore: World Scientific (2000). doi: 10.1142/3779

29. Akahtani B, Gulati V, Kilicman A. Application of sumudu transform in generalized fractional reaction-diffusion equation. Int J Appl Comput Math. (2016) 2:387-94. doi: 10.1007/s40819-015-0066-2

30. Garra R, Gorenflo R, Polito F, Tomovski Z. Hilfer-Prabhakar derivatives and some applications. Appl Math Comput. (2014) 242:576-89. doi: 10.1016/j.amc.2014.05.129

31. Panchal SK, Khandagale AD, Dole PV. Sumudu transform of HilferPrabhakar fractional derivatives with applications. In: Proceeding of Natural Conference on Recent Trends in Mathematics, Vol. 1. pp. 60-66.

32. Brockmann D, Sokolov IM. Levy flights in external force fields: from model to equations. Chem. Phys. (2002) 284:409-21. doi: 10.1016/S0301-0104(02)00671-7

Conflict of Interest Statement: The authors declare that the research was conducted in the absence of any commercial or financial relationships that could be construed as a potential conflict of interest.

Copyright (c) 2019 Gill, Singh and Singh. This is an open-access article distributed under the terms of the Creative Commons Attribution License (CC BY). The use, distribution or reproduction in other forums is permitted, provided the original author(s) and the copyright owner(s) are credited and that the original publication in this journal is cited, in accordance with accepted academic practice. No use, distribution or reproduction is permitted which does not comply with these terms. 